\title{
SARS-CoV-2 Infection in Pregnancy: A Case Report on the First Emergency Cesarean Section in Vietnam and Literature Review

\author{
Tran Dinh Vinh ${ }^{1^{*}}$, Vu Van Long ${ }^{2}$ and Pham Chi Kong ${ }^{3}$
}

${ }^{1}$ Department of Obstetrics and Gynecology, Da Nang Hospital for Women and Children, Da Nang City, Vietnam

${ }^{2}$ Department of Postpartum, Da Nang Hospital for Women and Children, Da Nang City, Vietnam

${ }^{3}$ Department of Delivery, Da Nang Hospital for Women and Children, Da Nang City, Vietnam

*Corresponding author: Tran Dinh Vinh, Department of Obstetrics, Director of Da Nang Hospital for Women and Children, 402 Le Van Hien Street, Ngu Hanh Son District, Da Nang City, Vietnam, Tel: (+84)023-639-57-777, Fax: 023-3957-779

\begin{abstract}
We report the first case of a pregnant woman infected with SARS-CoV-2 having an emergency cesarean section in Vietnam. This is the $569^{\text {th }}$ patient infected with SARS-CoV-2 in Vietnam and is one of the first two pregnant women infected with SARS-CoV-2 in Vietnam (along with patient number 495 who is 11 weeks pregnant). The patient has a history of treatment at Da Nang General Hospital (an outbreak in Da Nang City) due to urinary tract infection. The patient was discharged from the hospital on July 21, 2020. Due to epidemiological factors, screening for SARSCoV-2 should be conducted. Upon hospital admission, the woman was presented with 02 previous C-section scars, no fever, mild dry cough and no dyspnea. Patient still suffered burning and pain during urination. Patient still suffered burning and pain during urination. Normal white blood cell count. The RT-PCR test for SARS-CoV-2 in nasopharyngeal swab specimens showed positive results for 4 times (each was 2-5 days following the previous one) and one negative result after 12 days. After 2 weeks of follow-up, the pregnant women showed signs of labor and pain in the two previous $\mathrm{C}$-section scars and be indicated for cesarean section, giving birth to a baby girl, $3000 \mathrm{~g}$, IA 8/1'9/5' at 9:10 pm on August 15, 2020. After surgery, the baby and the mother were in the same room and the baby was breastfed. The postpartum process is stable. Both mother and baby were discharged from hospital on August 22, 2020 after 3 negative test results.
\end{abstract}

\section{Keywords}

COVID-19, Neonate, Pregnancy, SARS-CoV-2, Vertical transmission

\section{Introduction}

On March 11, the World Health Organization officially declared an outbreak of acute respiratory infections (COVID-19) caused by a new strain of the Corona virus (SARS-CoV-2) as a pandemic [1]. Vietnam reported the first case of SARS-CoV-2 infection on January 22, 2020 being a Chinese coming from Wuhan - where earliest case in the world was detected [2]. As of September 4, 2020, there were 26, 455, 964 cases recorded in the world and in Vietnam the number of infected people is 1046 [3].

Although the number of SARS-CoV-2 infections is increasing globally, evidence of the spread, incidence, and impact of SARS-CoV- 2 infection on both mother and child remains limited [4]. Due to physiological changes in the immune system and cardiovascular-respiratory system, pregnant women are considered to be at high risk of viral respiratory infections. Based on data from previous outbreaks of severe acute respiratory syndrome Coronavirus (SARS-CoV) and Middle East respiratory syndrome coronavirus (MERS-CoV), it has been found that pregnant women are more susceptible to adverse outcomes such as the need of intubation, intensive care, renal failure and death [5].

A meta-analysis by Allotey J, et al., including 77 studies $(13,118$ women infected with SARS-CoV-2 and 83,486 women of non-pregnant age of childbearing

Citation: Vinh TD, Long VV, Kong PC (2020) SARS-CoV-2 Infection in Pregnancy: A Case Report on the First Emergency Cesarean Section in Vietnam and Literature Review. Obstet Gynecol Cases Rev 7:177. doi.org/10.23937/2377-9004/1410177

Accepted: October 10, 2020: Published: October 12, 2020

Copyright: (C) 2020 Vinh TD, et al. This is an open-access article distributed under the terms of the Creative Commons Attribution License, which permits unrestricted use, distribution, and reproduction in any medium, provided the original author and source are credited. 
age) showed the incidence of SARS-CoV-2 infection in incoming women for examination or hospitalization for any reason is $10 \%$. The rates vary with sampling strategy: $7 \%$ for universal screened cases and $18 \%$ for symptomatic cases [6]. According to a meta-analysis and systematic review by Khalil A, et al., the most common clinical symptoms in pregnant women infected with SARSCoV-2 are cough $(71.4 \%, 95 \% \mathrm{Cl} 66.2-76.2 \%)$, fever. (63.3\%, 95\% Cl 54.7-71.2\%) and dyspnea $(34.4 \%, 95 \% \mathrm{Cl}$ 25.7-44.4\%) [7]. There is ample evidence that viral infections can cause serious complications during pregnancy. However, there still have been conflicting results on the effects of COVID-19 in pregnancy [8].

Currently, there is still no clear evidence for an optimal time to terminate a pregnancy, or whether vaginal delivery or cesarean section is safe in preventing mother-to-child transmission at birth [9]. The systematic review by Juan, et al. showed that, despite the increasing number of studies, there was no good quality evidence to draw conclusions to the severity of the disease and the disease-specific complications in pregnant women as well as problems of vertical transmission and complications in the infant and perinatal period [5].

Here, we report the first emergency cesarean section for a pregnant woman infected with SARS-CoV-2 in Vietnam. This is the $569^{\text {th }}$ patient infected with SARSCoV-2 in Vietnam and is one of the first two pregnant women infected with SARS-CoV-2 in Vietnam (along with patient No. 495 who is 11 weeks pregnant).

\section{Case Report}

35-year-old pregnant woman with 35 weeks of pregnancy was admitted to Hoa Vang District Field Hospital on August 1, 2020, transferred from Lien Chieu District Hospital since patient's screening test for SARS-CoV-2 was positive.

The patient has a history of treatment at Da Nang General Hospital (an outbreak in Da Nang City) due to urinary tract infection. The patient was discharged from the hospital on July 21, 2020. Due to epidemiological factors, screening test for SARS-CoV-2 was conducted.

Upon admission to Hoa Vang District Field Hospital, the woman's temperature was $37^{\circ} \mathrm{C}$ Blood pressure was $110 / 70$, breathing rate at 20 times/minute, she had a mild dry cough but no difficulty in breathing, the oxygen saturation was $97 \%$. Blood count test was normal (red blood cell $4.49 \times 10^{12} / \mathrm{L}$, Hematocrit $39.0 \%$, Hemoglobin $131 \mathrm{~g} / \mathrm{L}$, white blood cell count $5.5 \times 10^{9} / \mathrm{L}$, neutrophils $75.1 \%$, lymphocyte $22.1 \%$ ). The pregnancy ultrasound was normal. The RT-PCR technique for SARS-CoV-2 nasopharyngeal fluid was positive for 4 times (each was 2-5 days following the previous one) and one negative result after 12 days from the first test. According to the guidelines for diagnosis and treatment of acute respiratory infections caused by SARS-CoV-2 (COVID-19) of the Ministry of Health of Vietnam, this pregnant wom- an was classified as a mild-type COVID-19 patient. After 3 days of hospitalization, the cough symptoms disappeared. Due to symptoms of urinary tract infections, the patient was given an antibiotic (Zinnat $0.5 \mathrm{~g} \times 02$ tablets) day for 7 days). In addition, multivitamins were also given. During the hospital follow-up, the patient had no fever, no difficulty in breathing, and did not need oxygen support. Cardiotocography was measured every 3 days, ultrasound weekly. After 2 weeks of follow-up, the pregnant women showed signs of labor, had old wound pain and gestational age was 37 weeks, therefore she was indicated for cesarean section and gave birth to a 3000 $\mathrm{g}$ baby girl, IA $8 / 1^{\prime}-9 / 5^{\prime}$. The anesthesia method used was spinal anesthesia. During the cesarean section, the front of the uterus attached to the anterior abdominal wall, we had to separate the adherence so that the lower uterus was exposed. The operation was safe, there was no abnormal progress. Patients were using antibiotics (Zimdibiotic $1 \mathrm{~g} \times 02$ vials/day, slow intravenous injection, and Gentamycine $80 \mathrm{mg} \times 02$ ampoules/day intramuscularly) for 07 days. After surgery, the baby and the mother were in the same room and the baby was breastfed. The process of the post-cesarean section was stable. Both mother and baby were discharged from the hospital one week after the cesarean section, also after 3 negative tests. The total time for patients to be monitored and treated at the hospital was 22 days, of which 7 days post-cesarean section.

\section{Discussion}

Vietnam identified 270 cases of SARS-CoV-2 within 100 days after the first case on January 22, 2020, of which $60 \%$ were immigration, $43 \%$ were asymptomatic and no death case [10]. However, after 99 days without cases in the community since April 15, 2020, Vietnam found new cases coming from three hospitals in Da Nang City including Hospital C, Da Nang General Hospital and Da Nang Rehabilitation Hospital on July 16, 2020, and since then spread to provinces across the country. Da Nang city is identified as the epidemic center of this second outbreak. As of September 4, 2020, Vietnam recorded 1,046 cases of SARS-CoV-2, of which 35 were fatal [3].

Danang General Hospital is the largest general hospital in Da Nang city and one of the largest general hospitals in the Central-Central Highlands region of Vietnam. This was the first treatment site for patients infected with SARS-CoV-2 in Da Nang city. However, after assessing the hospital was a SARS-CoV-2 cluster in Da Nang, the City government and the Da Nang Department of Health decided to establish Hoa Vang District Field Hospital in the Hoa Vang district Health Center for treatment of SARS-CoV-2 infected patients, in order to limit the spread to other hospitals in the city. For this reason, the pregnant women in this report was transferred to the field hospital for follow-up and treatment, not to Da Nang Hospital for Women and Children-one of the leading hospitals in the Central Highlands region in the 
field of Obstetrics, Gynecology and Pediatrics. The Vietnam Ministry of Health and the Da Nang Department of Health have sent an official letter to Da Nang Hospital for Women and Children to make assessment and plan for the first pregnant woman in Vietnam infected with SARS-CoV-2 at Hoa Vang District Field Hospital [3].

Having epidemiological factor (a history of treatment at Danang General Hospital), the patient in this report was tested for SARS-CoV-2 which showed a positive result. In London, et al. study [11], 81 women were tested for SARS-CoV-2 due to positive screening (with clinical symptoms such as fever, cough including 60 women and exposure to COVID-19 patient including 21 pregnant women). The results showed that 58 cases were positive for SARS-CoV-2, accounting for $71.4 \%$, of which the rate of SARS-CoV-2 infection in the group tested due to exposure but no symptoms was $57.1 \%(12 / 21)$. Also in this study, the rate of SARS-CoV-2 infection in the mass screening group of 75 asymptomatic women was $13.3 \%$ (10/75) [11]. Another study by Fassett MJ, et al. of 3,923 women who were universal screened at the time of hospital admission for delivery showed a very low rate of SARS-CoV-2 infection $(17 / 3.923=0.43)$ and all in all cases there are no symptoms [12]. This suggests that screening for SARS-CoV-2 infection in pregnant women with risk factors (with clinical symptoms or exposure) is a reasonable strategy in middle-low-income countries such as Vietnam. In addition, obstetricians and midwives need to be very cautious during a pandemic when monitoring and treating for pregnant women of highrisk group.

The pregnant woman in this report had mild cough on admission, no fever, and no dyspnea. In addition, she also had urinary tract infections with burnings and pain during urination. According to a meta-analysis and systematic review by Gao Y, et al., the most common symptoms in pregnant women infected with SARSCoV-2 were fever (51\%; $95 \% \mathrm{Cl}, 0.35-0.67)$, cough (31\%; $95 \% \mathrm{Cl}, 0.23-0.39)$ and the proportion of patients with coexisting disorders is $33 \%(95 \% \mathrm{Cl}, 0.21-0.44)$. In addition, the rates of fever and cough in SARS-CoV-2-infected pregnant women were statistically significantly lower than those of non-pregnant women infected with SARS-CoV-2. This may be due to changes in the immune system in pregnant women. Currently, there is no evidence that pregnancy alleviates symptoms of the disease and more studies are needed to confirm this [13]. This result is similar to a new meta-analysis published in early September 2020: Pregnant women infected with SARS-CoV-2 have less symptoms of fever (OR $0.43,95 \%$ $\mathrm{Cl} 0.22-0.85$ ) and myalgia (OR $0.48,95 \% \mathrm{Cl} 0.45-0.51$ ) compared with non-pregnant women infected with SARS-CoV-2 [6]. Meanwhile, another meta-analysis by Matar, et al. showed the clinical characteristics of pregnant women infected with SARS-CoV-2 were not different from the group of non-pregnant women infected with SARS-CoV-2 [14]. According to these authors, due to limitations, better designed studies are needed to draw clearer conclusions about the clinical and subclinical characteristics of pregnant women infected with SARS-CoV-2 compared with the non-pregnant women with SARS-CoV-2 and the general population group. A meta-analysis showed that older mothers (OR1.78, 95\% $\mathrm{Cl} 1.25-2.55)$, high body mass index (OR $2.38,95 \% \mathrm{CI}$ 1.67-3.39), hypertension (OR 2.0, 95\% $\mathrm{Cl} 1.14-3.48$ ) and diabetes $(2.51,95 \% \mathrm{Cl} 1.31-4.80)$ are associated with severity of the disease in pregnancy period. Pre-existing maternal comorbidity are the risk factors for being in intensive care (OR 4.1, 95\% $\mathrm{Cl} 1.06-16.72)$ and invasive mechanical ventilation (OR 4.48, 95\% Cl 1.40-14.37) [6].

The patient is indicated for cesarean section for obstetric reasons (pain in the prior C-section) and spinal anesthesia is the method of anesthesia chosen. This is also consistent with the Guidance on temporary prevention and management of COVID-19 disease in pregnant women and infants by Vietnam Ministry of Health issued on March 21, 2020 [3]. According to Global interim guidance on coronavirus disease 2019 (COVID-19) during pregnancy and puerperium from The International Federation of Gynaecology and Obstetrics (FIGO), COVID-19 infection is not an indication for termination of pregnancy. The timing and method of delivery should be individualized, depending mainly on the clinical status, gestational age and fetal status. The anesthesia method in our case is also consistent with the recommendations of FIGO and the Obstetric Anesthesia Associations [15].

The impact of SARS-CoV-2 infection on pregnancy outcomes is of concern. There is ample evidence that maternal viral infection can lead to adverse pregnancy outcomes due to the effect of the causative agent to the woman. Studies in pregnant women infected with other coronavirus strains have shown that viral infection increases the risk of maternal death, miscarriage, intrauterine growth retardation, and preterm birth. On the other hand, the influenza vaccine has been shown to reduce the risk of preterm birth in pregnant women exposed to influenza. One of the possible explanations for these adverse outcomes can be attributed to the induction of the most severe inflammatory state that occurs as a result of viral infection. The normal physiological changes in the third trimester of pregnancy create pro-inflammatory state so that a woman's body prepares for the beginning of the parturition. SARS-CoV-2 infection is associated with increased cytokine production and this causes the immune system to change similarly to the third trimester, causing uterine contractions, amniotic fluid rupture, and birth $[9,16]$. The systematic review of Turan O, et al. [17] showed that the rates of maternal mortality, stillbirth, and neonatal mortality were $1.6 \%, 1.4 \%$ and $1.0 \%$, respectively. Elderly mother, obesity, diabetes, increased D-dimer and interleukin- 6 levels were predictors of adverse outcomes. The rate of preterm birth was $33.7 \%$. Most women under- 
went cesarean section without clear indication. $2 \%$ of infants were tested positive for SARS-CoV-2 and progressed to pneumonia within 48 hours of birth [17]. According to a meta-analysis and systematic review by Di Mascio, et al., SARS-CoV-2 infection resulted in preterm birth $(24.3 \%, 95 \% \mathrm{Cl} 12.5-38.6)$ for gestational age $<37$ weeks, and $21 \%$ (95\% Cl 12.5-32.9) for gestational age < 34 weeks, preeclampsia $(16.2 \%$, 95\% Cl 4.2 $34.1)$ and cesarean section $(83,9 \%, 95 \% \mathrm{Cl} 73.8-91.9)$ [18]. The risk of admission to neonatal unit for infants of mothers infected with SARS-CoV-2 was three times higher (OR 3.13, 95\% Cl 2.05-4.78) compared to children whose mothers were not infected with SARS-CoV-2 [6]. To our knowledge, as of early September 2020, there have been 07 meta-analyzes and systematic reviews of pregnancy outcomes in SARS-CoV-2 infected pregnant women published in English. Results of 06 meta-analyzes and systematic reviews all showed that the rate of preterm birth and cesarean section in pregnant women infected with SARS-CoV-2 was higher than in women without SARS-CoV-2 $[6,7,13,14,18,19]$, meanwhile, 01 meta-analysis by Melo, et al. had the opposite result, that COVID-19 infection in pregnant women might not associated with preterm birth [9]. However, the authors of these meta-analyzes and systematic reviews all argue that cautions should be taken when interpreting the results due to limitations (i.e most of studies were retrospective studies and case reports; and lack of high quality evidence).

Identification of vertical transmission from mother to fetus and child has an important role in developing principles of obstetric care in pregnant women infected with SARS-CoV-2. In addition, the long-term health effects of exposure to the virus during pregnancy in infants and young children are unknown [20]. The infant of the mother in this report had three times been tested for SARS-CoV-2 and found negative. Vertical transmission has been identified for many viruses and may cause perinatal adverse outcomes such as miscarriage, delayed fetal growth, premature birth and stillbirth. Several vertical pathways include intrauterine transmission, transmission during delivery, breast milk transmission, and postpartum exposure. Among these pathways, intrauterine transmission is the least likely to be controlled and treated. This is the most important pathway of mother-to-child transmission and affects fetus and infant [21]. A systematic review by Elshafeey, et al. [22] showed that 4 out of 256 infants born to a mother infected with SARS-CoV-2 had a positive RT-PCR (1.6\%) result and had mild symptoms. However, samples from cord blood, placenta and amniotic fluid of these four infants were negative [22]. A meta-analysis and systematic review by Kotlyar A, et al. [23] showed that the vertical transmission rate was 3.2\% (95\% Cl 2.2$4.3 \%)$. The authors concluded that vertical transmission of SARS-CoV-2 may occur in a small number of mothers infected with COVID-19 during the third trimester of pregnancy. This ratio is equivalent to other agents causing congenital infection. In addition, due to limited data in the first trimester of pregnancy, vertical transmission in the first trimester is not yet assessed, nor the risk of death and subsequent morbidity in these cases. However, the authors noted that caution must be exercised in interpreting this vertical transmission as one of the limitations of this meta-analysis was that there was a significant difference in quality between studies and between reported data [23]. According to a systematic review by Yang $Z$, et al., at present, there is no direct evidence of vertical transmission in the uterus. RT-PCR tests for amniotic fluid, placenta and cord blood are needed to confirm the possibility of vertical transmission in the uterus. For pregnant women infected with SARS-CoV-2 in the first and second trimesters, studies that focus on long-term outcomes are needed [21].

The infant in our report was breastfed and roomed with her mother postpartum after discussion with the infant's family. According to Guidelines of The International Federation of Gynecology and Obstetrics and other organizations (World Health Organization, American College of Obstetricians and Gynecologists and Royal College of Obstetricians and Gynecologists), there is currently insufficient evidence regarding the safety of breastfeeding and the need for mother/baby separation. If the mother is severely or critically ill, separating mother and child is the optimal option. If the patient is asymptomatic or has mild symptoms, breastfeeding and rooming-in can be considered [15].

\section{Conclusion}

SARS-CoV-2 infection was not yet in the newborns born to the pregnant women with COVID-19. Pregnant women infected SARS-CoV-2 should be followed and managed by multidisciplinary team.

\section{Conflict of Interest}

The authors declare that they have no conflict of interest regarding the publication of this case report.

\section{Funding}

This research did not receive any specific grant from funding agencies in the public, commercial, or not-forprofit sectors.

\section{Statement of Equal Authors' Contribution}

I confirm that all authors have contributed significantly, and that all authors agree with the content of the manuscript.

\section{Patient Consent}

Obtained.

\section{Disclosure Statement}

The author(s) report(s) no conflicts of interest. 


\section{Financial Support}

No financial sources of support were used to compose this article.

\section{References}

1. World Health Organization (2020) Rolling updates on coronavirus disease (covid-19).

2. Phan LT, Nguyen TV, Luong QC, Thinh $\vee$ Nguyen, Hieu T Nguyen, et al. (2020) Importation and human-to-human transmission of a novel coronavirus in Vietnam. N Engl J Med 382: 872-874.

3. Vietnam Ministry of Health (2020) COVID-19 pandemic.

4. Knight M, Bunch K, Vousden N, Edward Mo, Nigel S, et al. (2020) Characteristics and outcomes of pregnant women admitted to hospital with confirmed SARS-CoV-2 infection in UK: National population based cohort study. BMJ 369.

5. Juan J, Gil MM, Rong Z, Zhang Y, Yang H, et al. (2020) Effect of coronavirus disease 2019 (COVID-19) on maternal, perinatal and neonatal outcome: Systematic review. Ultrasound Obstet Gynecol 56: 15-27.

6. Allotey J, Stallings E, Bonet M, Yap M, Chatterjee S, et al. (2020) Clinical manifestations, risk factors, and maternal and perinatal outcomes of coronavirus disease 2019 in pregnancy: Living systematic review and meta-analysis. BMJ 370 .

7. A Khalil, Erkan Kalafat, Can Benlioglu, Pat O'Brien, Edward Morris, et al. (2020) SARS-CoV-2 infection in pregnancy: A systematic review and meta-analysis of clinical features and pregnancy outcomes. E Clinical Medicine.

8. Sahin D, Tanacan A, Erol SA, Ali T Anuk, Elif GY Eyi, et al. (2020) A pandemic center's experience of managing pregnant women with COVID-19 infection in Turkey: A prospective cohort study. Int J Gynaecol Obstet.

9. Melo Gessyca Cavalcante de, Araujo Karina Conceicao Gomes Machado de (2020) COVID-19 infection in pregnant women, preterm delivery, birth weight, and vertical transmission: A systematic review and meta-analysis. Cadernos de Saude Publica 36.

10. Pham QT, Rabaa MA, Duong HL, Quang Tan Dang, Dai Quang Tran, et al. (2020) The first 100 days of SARSCoV-2 control in Vietnam. Clin Infect Dis.

11. London V, McLaren R Jr, Atallah F, Catherine C, Sandra Mc, et al. (2020) The relationship between status at presentation and outcomes among pregnant women with COVID-19. Am J Perinatol 37: 991-994.
12. Fassett MJ, Lurvey LD, Yasumura L, Marielle Nguyen, Joseph J Coll, et al. (2020) Universal SARS-Cov-2 screening in women admitted for delivery in a large managed care organization. Am J Perinatol.

13. Gao YJ, Ye L, Zhang JS, Yang-xue Y, Min L, et al. (2020) Clinical features and outcomes of pregnant women with COVID-19: A systematic review and meta-analysis. BMC Infect Dis 20: 564.

14. Matar R, Alrahmani L, Monzer N, Labib G, Elie B, et al. (2020) Clinical presentation and outcomes of pregnant women with COVID-19: A Systematic Review and Meta-Analysis. Clin Infect Dis.

15. Poon LC, Yang H, Kapur A, Nir M, Blami D, et al. (2020) Global interim guidance on coronavirus disease 2019 (COVID-19) during pregnancy and puerperium from FIGO and allied partners: Information for healthcare professionals. Int J Gynaecol Obstet 149: 273-286.

16. Narang K, Enning EA, Gunaratne, Eniola R Ibirogba, Ayssa Teles A Trad, et al. (2020) SARS-CoV-2 infection and COVID-19 during pregnancy: A multidisciplinary review. Mayo Clin Proc 95: 1750-1765.

17. Turan O, Hakim A, Dashraath $P$, Jeslyn WJL, Wright A, et al. (2020) Clinical characteristics, prognostic factors, and maternal and neonatal outcomes of SARS-CoV-2 infection among hospitalized pregnant women: A systematic review. Int J Gynaecol Obstet.

18. Di Mascio D, Khalil A, Saccone G, Giuseppe R, Danilo B, et al. (2020) Outcome of coronavirus spectrum infections (SARS, MERS, COVID-19) during pregnancy: A systematic review and meta-analysis. Am J Obstet Gynecol MFM.

19. Capobianco G, Saderi L, Aliberti S, Michele M, Andrea P, et al. (2020) COVID-19 in pregnant women: A systematic review and meta-analysis. Eur J Obstet Gynecol Reprod Biol 252: 543-558.

20. Zeng Y, Lin L, Yan Q, Wei W, Bing X, et al. (2020) Update on clinical outcomes of women with COVID-19 during pregnancy. Int J Gynaecol Obstet 150: 264-266.

21. Yang Z, Liu Y (2020) Vertical transmission of severe acute respiratory syndrome coronavirus 2: A systematic review. Am J Perinatol 37: 1055-1060.

22. Elshafeey F, Magdi R, Hindi N, Mohamed E, Nourhan F, et al. (2020) A systematic scoping review of COVID-19 during pregnancy and childbirth. Int J Gynaecol Obstet 150: 47-52.

23. Kotlyar A, Grechukhina O, Chen A, Popkhadze S, Grimshaw A, et al. (2020) Vertical transmission of COVID-19: A systematic review and meta-analysis. Am J Obstet Gynecol. 